\title{
Long-QT syndrome: to be or not to be iatrogenesis - a case report
}

\author{
Dragoş Traian Marius Marcu ${ }^{1,2}$, Alina Hohaci ${ }^{1}$, Mădălina Ioana Chiorescu ${ }^{1,2}$, Carmen \\ Elena Pleșoianu ${ }^{1,2}$, Cristian Stătescu ${ }^{1,2}$, Mircea Balasanian ${ }^{1,2}$, Liviu Macovei*1,2, \\ Cătălina Arsenescu-Georgescu ${ }^{1,2}$
}

1"Prof. Dr. George I.M. Georgescu" Institute of Cardiovascular Diseases, lasi, Romania, "”Grigore T. Popa" University of Medicine and Pharmacy, lasi, Romania

\begin{abstract}
Long-QT syndrome is a congenital or acquired disorder of the heart's electrical activity, inducing delayed repolarization that can cause ventricular arrhythmias and sudden cardiac death. Acquired forms can occur as a result of various drugs and given the fact that most patients have multiple comorbidities, the risk of polymedication and iatrogenesis is increasingly high. We present the case of a 60 -year-old female admitted to the Cardiology Department for an arrhythmic storm, associating QT interval prolongation. The patient had a known history of atrial fibrillation and was undergoing antiarrhythmic therapy with flecainide. The hypothesis of a drug-induced long-QT syndrome was raised. Multiple ventricular arrhythmias requiring defibrillation occurred and intubation with mechanical ventilation was needed, followed by temporary ventricular pacing. We decided the discontinuation of flecainide therapy but over the next days, the patient presented persistent QT interval prolongation, multiple recurrences of ventricular arrhythmias and recurrent paroxysmal atrial fibrillation. In this context, the initial hypothesis of a flecainide-induced long QT syndrome was invalidated and given the persistent QT interval prolongation (QTc > $480 \mathrm{~ms}$ ); the hypothesis of a congenital long-QT syndrome was raised. An implantable cardioverter defibrillator implantation was performed. The ECG holter monitoring after implantation showed the disappearance of ventricular arrhythmias, with favorable clinical evolution.
\end{abstract}

Keywords: long-QT syndrome, iatrogenesis, arrhythmic storm, ventricular arrhythmias

\section{Introduction}

Long-QT syndrome (LQTS) is a congenital or acquired disorder of the heart's electrical activity, inducing delayed repolarization that can cause ventricular arrhythmias and sudden cardiac death. The congenital forms - JervellLange-Nielsen syndrome and Romano-Ward syndrome - are caused by inherited channelopathies, most frequently due to mutations of the KCNQ1, KCNH2 and SCN5A genes.

Received: February 2017; Accepted after review: June 2017; Published: June 2017

${ }^{*}$ Corresponding author: Macovei Liviu, MD, PhD, "Grigore T. Popa" University of Medicine and Pharmacy, 16, Universitatii Street, 700115, lasi, Romania.

E-mail: liviughemacovei@gmail.com
Acquired forms can occur as a result of various drugs and may also have an underlying genetic predisposition [1]. Nowadays, polymedication is a given fact, leading to increased risk of iatrogenesis and making the differential diagnosis between acquired and congenital LQTS quite difficult. In these conditions, patients undergoing antiarrhythmic therapy with drugs known to prolong the QT interval pose a real diagnostic challenge. Furthermore, given the different therapeutic approach in the two cases, an unnoticed congenital LQTS, can lead to fatal consequences. 


\section{Case report}

We present the case of a 60-year old female admitted to the Cardiology Department for multiple recurrences of ventricular arrhythmias (Figures $1 \mathrm{a}, 1 \mathrm{~b}$ and $1 \mathrm{c}$ ), resulting in an arrhythmic storm, associating QT interval prolongation. The patient had a known history of paroxysmal atrial fibrillation (AF), and was undergoing prophylactic treatment with flecainide and also ramiprilum, apixabanum and rosuvastatinum.
It should be noted that, during the last 3 years, the patient underwent antiarrhythmic treatment with amiodarone, followed by propafenone and, since last year, $200 \mathrm{mg}$ daily of flecainide. No history of other QT interval prolonging drugs (antibiotics, antipsychotics or antihistamines) could be found.

During initial assessment in the emergency room, the patient was conscious and the vital signs showed as follows: blood pressure (BP) - 120/70 $\mathrm{mmHg}$, heart rate $90 / \mathrm{min}$, respiratory rate $-18 / \mathrm{min}, \mathrm{O}_{2}$ saturation $-96 \%$ and a temperature of $36,5^{\circ} \mathrm{C}$.

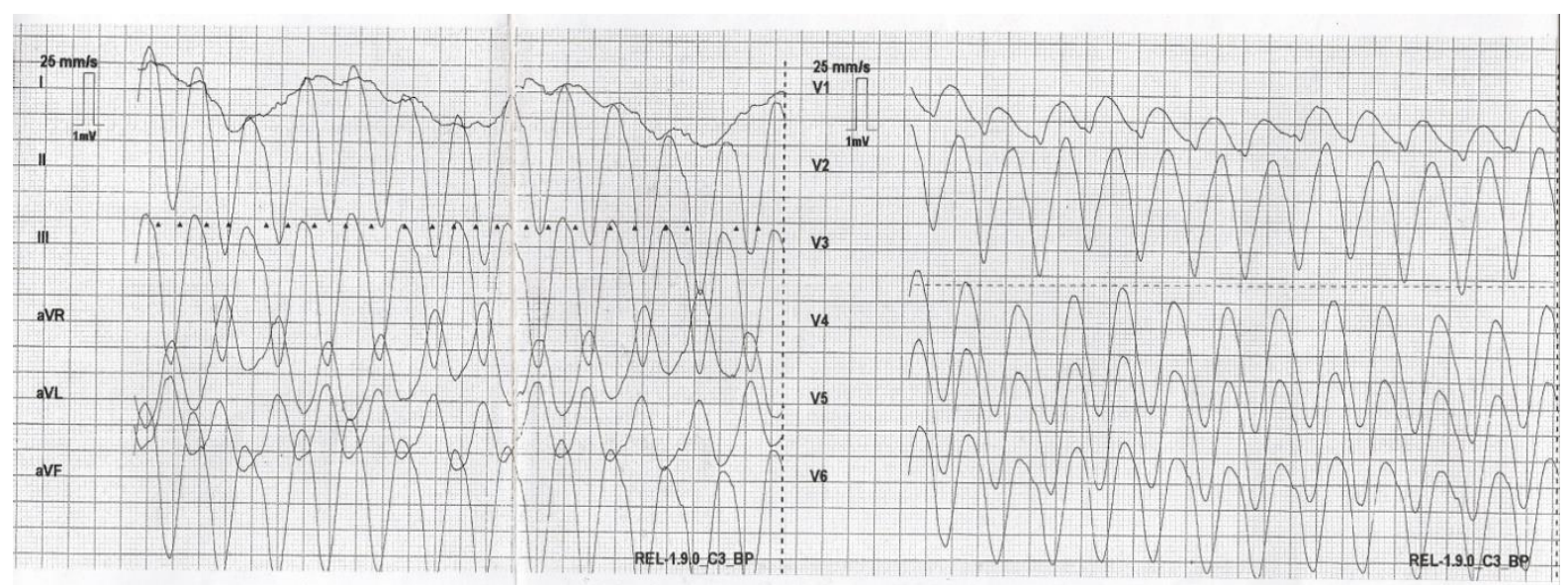

Fig. 1a. 12 lead ECG shown monomorphic ventricular tachycardia: wide QRS tachycardia with negative concordance, depicting the absence of an RS complex in all precordial leads thus meeting the Brugada criteria for ventricular tachycardia [2]
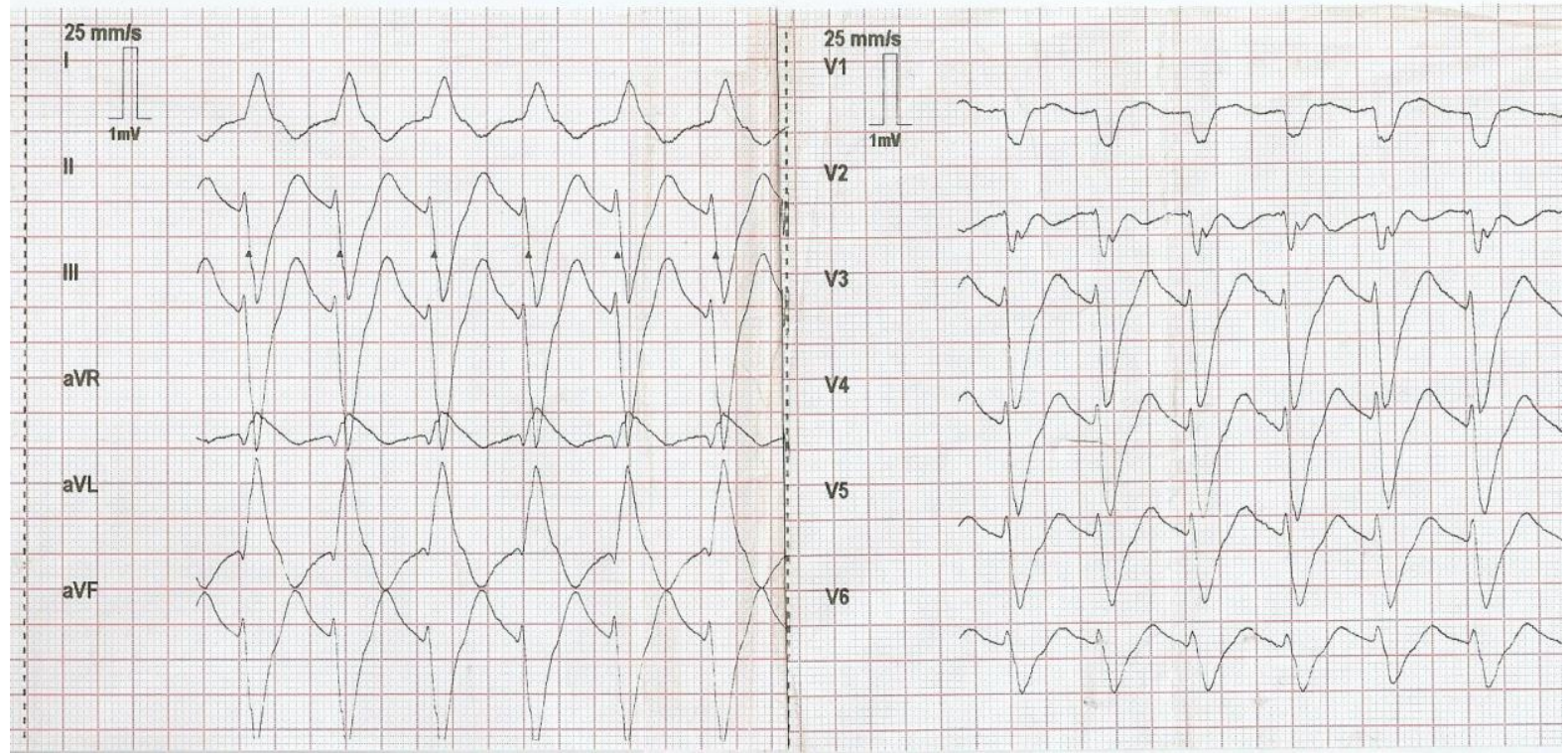

Fig. 1b. 12 lead ECG. Monomorphic ventricular tachycardia - second episode: wide QRS complex tachycardia, meeting the criteria for left bundle branch block configuration ventricular tachycardia, QRS width over $160 \mathrm{ms,}$ notching of the $S$ wave and also initial q wave $>40 \mathrm{~ms}$, in aVR $[3,4]$ 


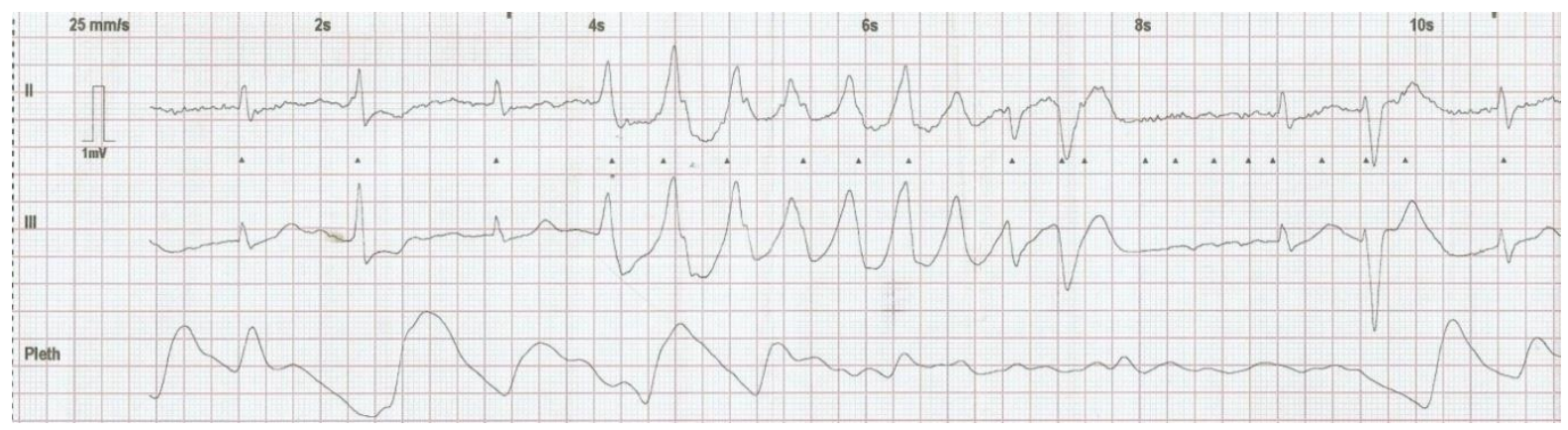

Fig. 1c. Two leads ECG monitor strip showing polymorphic ventricular tachycardia. QT interval is about $480 \mathrm{~ms}$, with a QTc of $507 \mathrm{~ms}$

The 12 leads ECG on admission, showed ventricular bigeminy followed by an episode of polymorphic ventricular tachycardia (VT) torsades de pointes (TdP) (Figure 2). Immediate measures were taken, using intravenous magnesium and lidocaine but without success. Over 20 sustained episodes of ventricular tachycardia requiring defibrillation occurred.

Blood tests revealed an inflammatory syndrome and mild anemia, without any electrolyte disorders or renal impairment.

The transthoracic echocardiography noted only a mild mitral regurgitation, with a left ventricular ejection fraction (LVEF) of $60 \%$, without regional wall motion abnormalities.

Given the situation, we decided that intubation and mechanical ventilation were needed, followed by temporary ventricular pacing (Figure 3 ).

Considering all above mentioned data and the fact the patient underwent a coronary angiography 4 months prior to current admission, that showed normal flow through the coronary arteries, thus ruling out an ischemic etiology, the hypothesis of a druginduced long-QT syndrome (LQTS) was raised

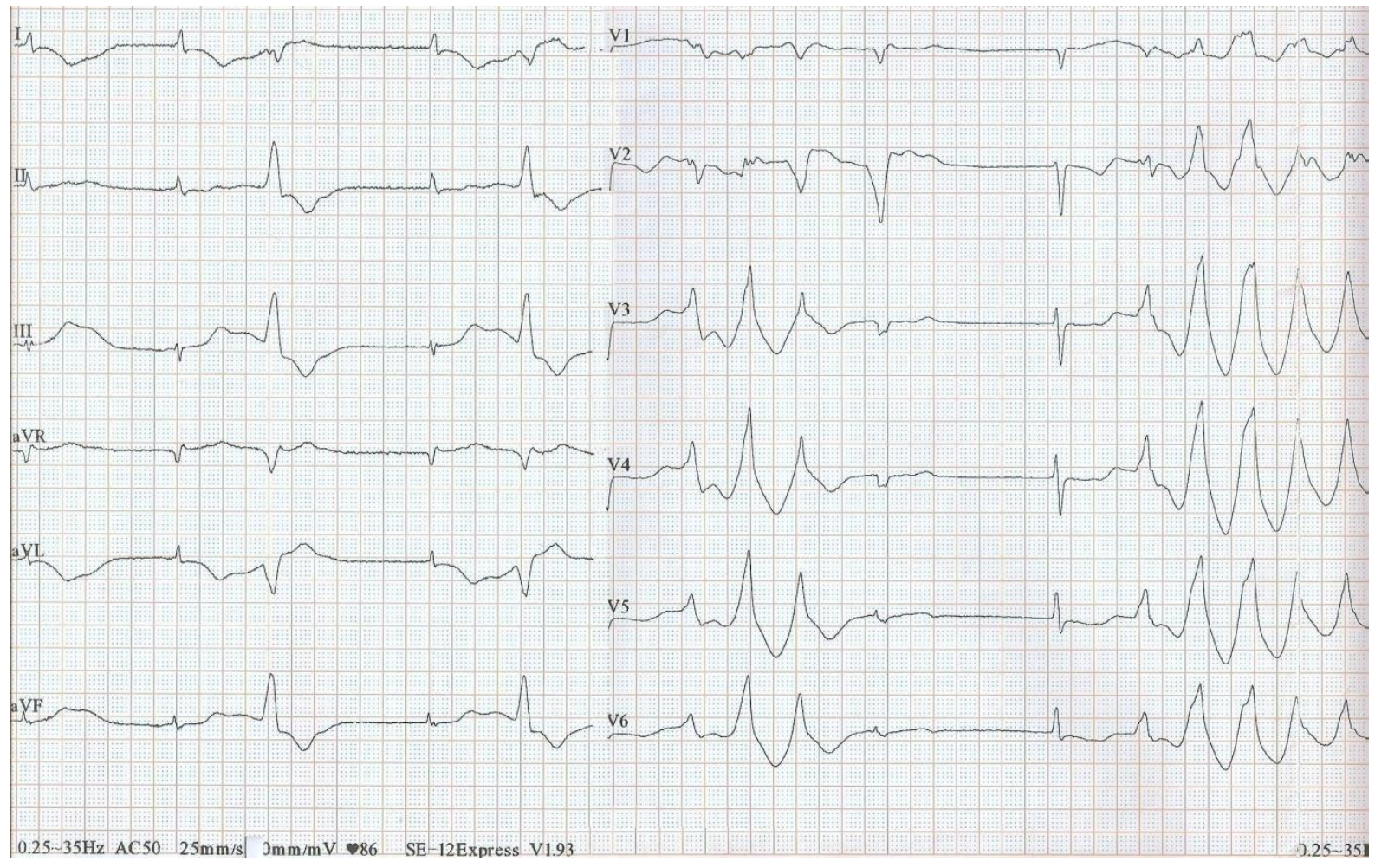

Fig. 2. 12 lead ECG. Ventricular bigeminy followed by a ventricular triplet and TdP. QT interval is about $560 \mathrm{ms,}$ with a QTc of $501 \mathrm{~ms}$ 
At this point, we decided the discontinuation of flecainide therapy while continuing the antiarrhythmic therapy with lidocaine and adding a beta blocker. On the next day, endotracheal extubation was performed along with an echocardiographic reevaluation that showed severe left ventricular dysfunction and a LVEF of only $20 \%$.

Over the next few days, the patient presented persistent LQTS, multiple recurrences of ventricular arrhythmias and recurrent paroxysmal $\mathrm{AF}$, requiring increased doses of beta blocker. In this context, our initial hypothesis of a flecainide-induced LQTS was invalidated. Given the persistent QT interval prolongation (QTc > $480 \mathrm{~ms}$ ), the hypothesis of a congenital LQTS was raised. Confirmatory genetic testing was recommended.
In accordance with the 2015 ESC Guidelines for the management of patients with ventricular arrhythmias and the prevention of sudden cardiac death, implantable cardioverter defibrillator (ICD) implantation was performed (Figure 4), allowing adequate doses of antiarrhythmic drugs.

ECG holter monitoring afterwards showed the disappearance of ventricular arrhythmias, with favorable clinical evolution. LVEF underwent a significant increase up to $40 \%$. In these circumstances we considered the initial LVEF impairment as a consequence of repeated resuscitation. Given the fact that amiodarone, propafenone, flecainide and beta blockers were previously used but with no success, the patient was discharged under antiarrhythmic therapy with sotalol and oral anticoagulation therapy with apixaban

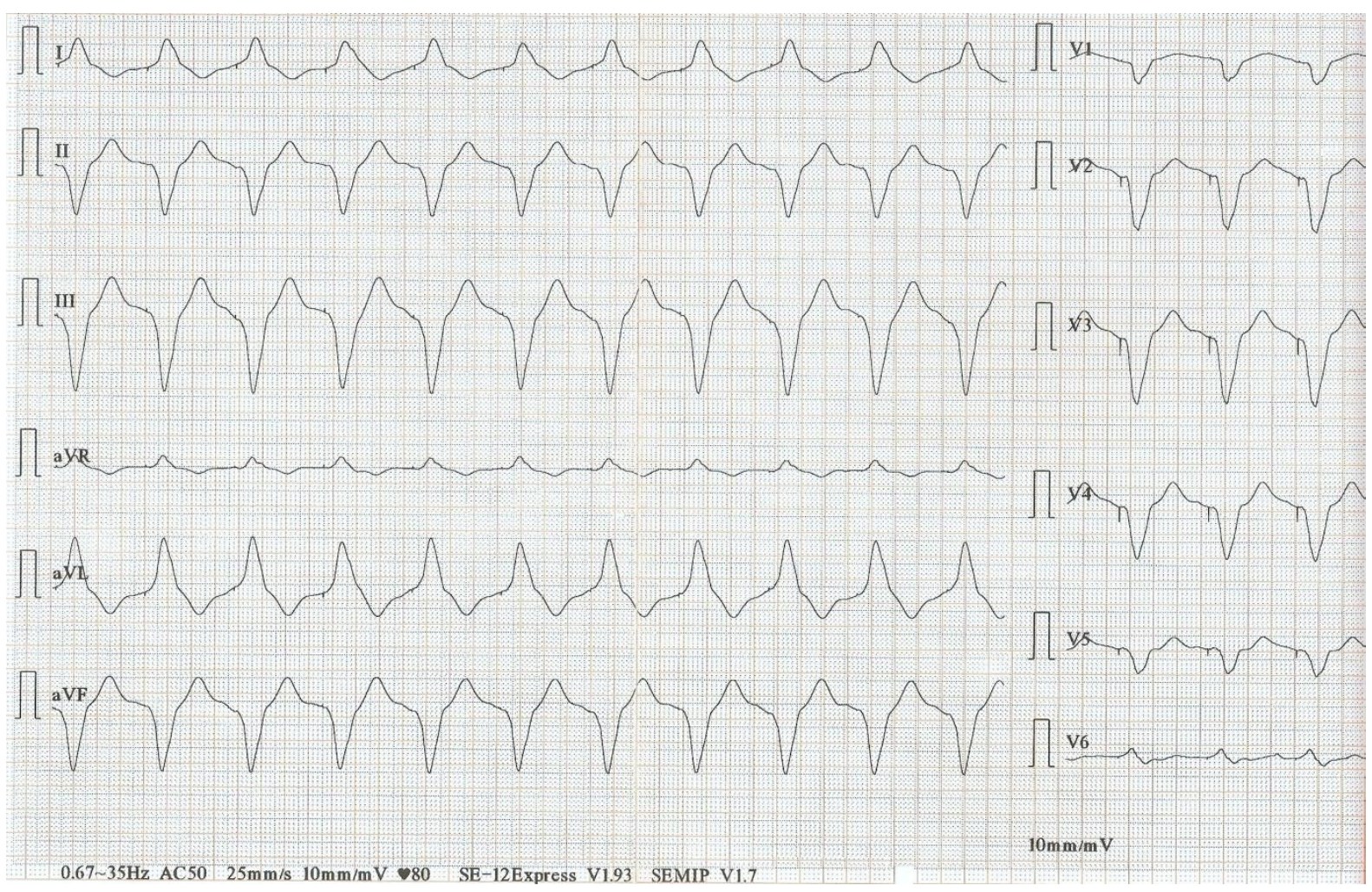

Fig. 3. 12 lead ECG after temporary ventricular pacing, depicting ventricular paced rhythm, pacing spikes visible before each QRS complex (especially in V2-V5) 


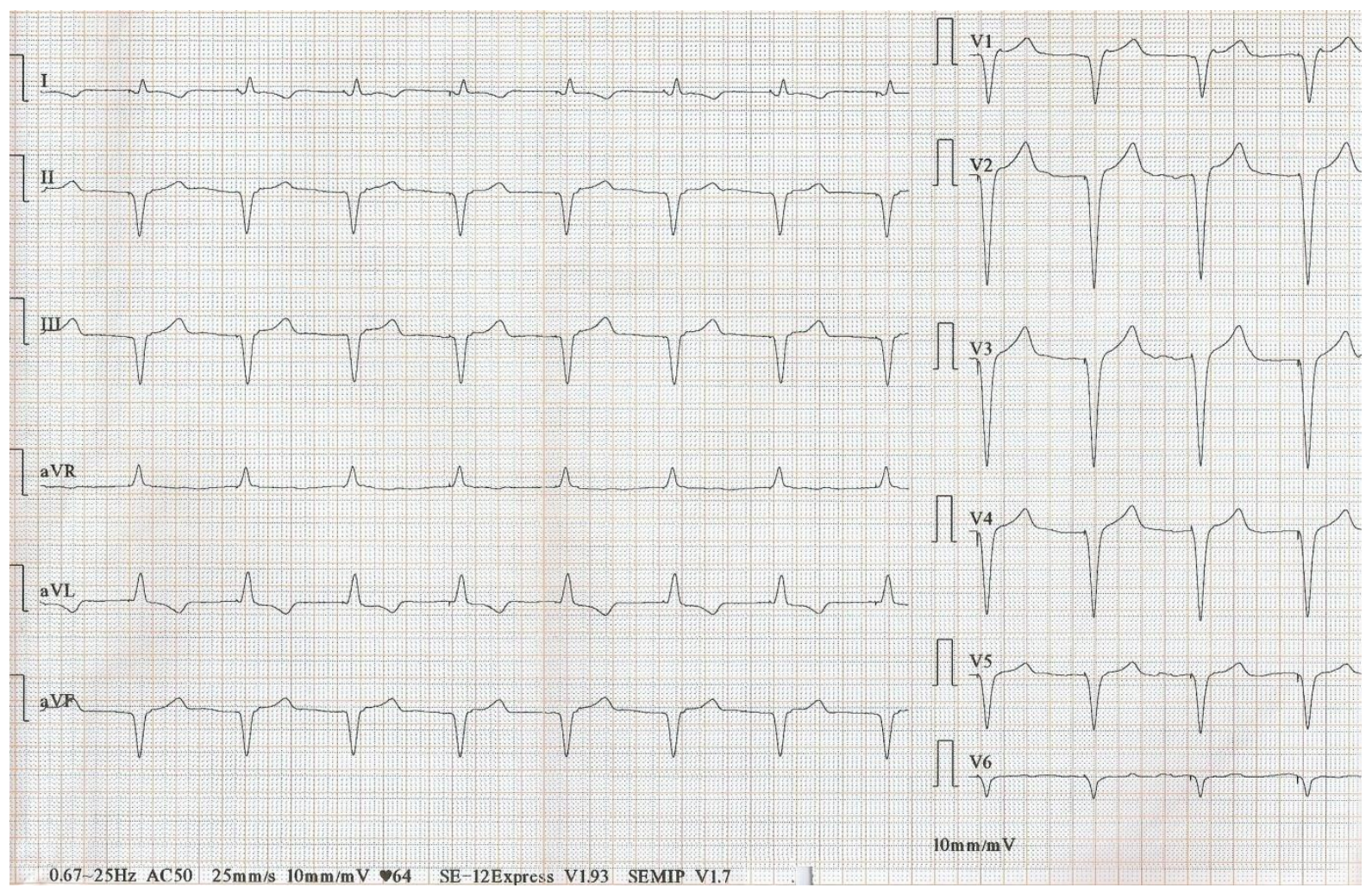

Fig. 4. 12 lead ECG after ICD implantation, depicting ventricular paced rhythm, with small pacing spikes before the QRS complex (bipolar lead)

\section{Discussions}

In the course of time, many drugs have been proved to exert a pro-arrhythmic effect on the heart. Most drug-induced ventricular arrhythmias occur due to the effect of prolonging ventricular repolarization that certain drugs have, leading to the occurrence of polymorphic VT. Among them, class IC antiarrhythmics and other anti-arrhythmics such as amiodarone have a leading role [1]. Most commonly, these rhythm disorders occur in patients with certain features: female, underlying structural heart disease, especially coronary artery disease, left ventricular dysfunction, and electrolyte disturbance [5, 6].

Drug-induced ventricular arrhythmias are rare and unpredictable events, with lifethreatening consequences. The main type of ventricular arrhythmia that should be considered is TdP, caused by QT interval prolongation. In this case, intravenous magnesium is the initial treatment of choice, along temporary ventricular pacing. Also, as shown before, lidocaine can be tried though, it should be noted that it is more effective in patients with coronary artery disease, compared to non-ischemic patients [1].

We reported a case of arrhythmic storm occurred in a female patient with a known history of $\mathrm{AF}$, undergoing treatment with flecainide. Flecainide is a class IC antiarrhythmic - potent sodium channel blocker, known to prolong the QT interval, most frequently after myocardial infarction [6].

It is important to highlight that, at this moment, with few exceptions, we do not possess a specific algorithm for determining whether a certain arrhythmia is iatrogenic or not. Because of this, sometimes, the needed course of action may be delayed until a reversible cause can be ruled out. To further complicate the issue, drug-induced QT interval prolongation involves molecular disorders targeting the same ionic channels as congenital LQTS. The severity of the disorder is not correlated with the drug's bioavailability, pharmacodynamics, and pharmacokinetics but with drug-drug interactions and patient dependent factors. 
Currently, gene study becomes particularly important in identifying genetic predisposition for TdP occurrence, following exposure to a particular drug. The human ether-a-go-go related gene (HERG) represents the main target of these studies due to its role in coding $\mathrm{KCNH} 2$ ion channel - the molecular substrate of QT prolongation [8].

Efforts are being undertaken to develop a comprehensive, regularly updated list of drugs known to prolong the QT interval, as to improve patients' management [9].

\section{Conclusions}

We presented a case in which iatrogenesis and genetics joined hands to form a fearful combination. Although each of these possibilities weren't totally unexpected, their summed effect gave birth to a rare and peculiar condition that posed important diagnostic and therapeutic issues. Currently, drug-induced arrhythmias are a challenge for clinicians from a wide range of specialties, not limited at all at being exclusive prerogatives of cardiology. This case highlights the issue posed by iatrogenesis in the current world. In

\section{References}

1. Mann DL, Zipes DP, Libby $\mathrm{P}$, Bonow RO, Braunwald E. Braunwald's Heart Disease - a textbook of cardiovascular medicine, 10th edition. Philadelphia: Elsevier Saunders, 2015.

2. Brugada $P$, Brugada $J$, Mont $L$ et al. $A$ new approach to the differential diagnosis of a regular tachycardia with wide QRS complex. Circulation 1991; 83:1649.

3. Wellens HJ, Bar FW, Lie KI. The value of the electroacardiogram in the differential diagnosis of a tachycardia with a widened QRS compex. Am J Med 1978; 64:27-33.

4. Vereckei A, Duray G, Szenasi G et al. New algorithm using only lead aVR for differential diagnosis of wide QRS complex tachycardia. Hearth Rythm 2008; 5:89-98.

5. Heist EK, Ruskin NJ. Drug-induced arrhythmia. Circulation 2010; 122:1426-1435. patients with subclinical mutations in the KCNQ1 or KCNH2 genes, certain antiarrhythmics may be the trigger for LQTS. Given the lethal potential of this syndrome, the best therapeutic approach should be a preventive one. However, the lack of a specific algorithm to identify high-risk patients makes primary prevention troublesome. In this context, accurately determining whether a certain arrhythmia is iatrogenic or not, is the best course of action and further efforts must be directed towards understanding and correctly diagnosing these life-threatening events.

\section{Consent}

Written informed consent was obtained from the patient for publication of this case report and accompanying images. A copy of the written consent is available for review by the Editor-in-Chief of this journal.

\section{Competing interests}

The authors declare that they have no competing interests.
6. Tinsdale JE, Miller DA. Drug-Induced Diseases - Prevention, Detection and Management, 2nd edition. Bethesda: American Society of HealthSystem Pharmacists, 2010.

7. Priori SG, Blomstrom-Lundqvist C, Mazzanti A, et al. 2015 ESC Guidelines for the management of patients with ventricular arrhythmias and the prevention of sudden cardiac death. Eur Heart J 2015; 36:27932867.

8. Konstantopoulou A, Tsikrikas S, Asvestas D, Korantzopoulos P, Letsas KP. Mechanisms of drug-induced proarrhythmia in clinical practice. World J Cardiol 2013; 5(6):175-185.

9. Woosley RL, Romero KA. QTdrug List [https://crediblemeds.org/ available at 01.31.2017]. 